\title{
ON THE SCHWARZ ALTERNATING METHOD FOR EIGENVALUE PROBLEMS
}

\author{
S.Y. MALIASSOV*
}

\begin{abstract}
In this paper an analogue of the Schwarz alternating method is considered to find a minimal eigenvalue and its corresponding eigenvector of generalized symmetric eigenvalue problem. The technique suggested is based on decomposition of the original domain into overlapping subdomains and on consideration of local eigenvalue problems in subdomains. Both multiplicative and additive variants of the method are constructed and studied.

It is shown that a discretization of the multiplicative variant of the Schwarz method is equivalent to the block coordinate relaxation method. An additive variant of the method is suitable for realization on parallel architecture.
\end{abstract}

Key words. Eigenvalue problem, Schwarz method, domain decomposition method.

AMS subject classifications. 65N25, 65N55, 65F15.

In this paper we propose an analogue of the Schwarz alternating method to evaluate the principal eigenvalue and its corresponding eigenfunction of a second order symmetric elliptic operator in a bounded domain in $\mathbb{R}^{d}$. The technique suggested is based on decomposition of the original domain into overlapping subdomains and on consideration of local eigenvalue problems in subspaces connected with these subdomains.

Domain decomposition methods (DD) are powerful techniques for solving boundary value problems. Recently, DD algorithms have become increasingly popular because they take full advantage of modern parallel computing technology. Although there are many papers on domain decomposition for the linear applications (see, e.g., [13, 5, 9, 1]), there are relatively few results on application of DD methods for eigenvalue problems.

One of the approaches is presented in [11] where an approximation to the principal eigenpair is computed by solving a sequence of linear problems in the subdomains. Several other domain decomposition methods also using linearization were proposed in $[10,15]$. These works are based on a nonoverlapping partitioning of the computational domain and using some iterative techniques for the Schur complement of the block corresponding to the interface variables. Another way to apply domain decomposition idea to eigenvalue problem is a divide-and-conquer method proposed in [4]. The authors introduced a parallel algorithm for computing all the eigenpairs of the symmetric and positive definite matrix, first, parallelizing the Householder transformation and, then, providing the multilevel parallel method of solving eigenvalue problem for three-diagonal symmetric matrix.

A different approach was presented by the author in the previous work [12] where only a multiplicative algorithm and its discretization were described with some limiting assumptions. In that algorithm an approximation to the principal eigenpair is computed by solving sequentially a series of minimization problems for the Rayleigh quotient in the subdomains. On the algebraic level this method

* Institute for Mathematics and Its Applications, 514 Vincent Hall, 206 Church Str. S.E., University of Minnesota, Minneapolis, MN 55455 (malyasov@ima.umn.edu). 
is equivalent to the one developed in [8] and can be considered as a generalization of the block coordinate relaxation applied directly for a matrix eigenvalue problem [6]. An important feature of this approach is that the subspace problems are also generalized eigenvalue problems, which allows to apply the algorithm recursively. The recursive implementation of this method was proposed in [2], where based on [12] and [8] the authors presented multilevel algorithm of optimal complexity. Due to a minor flaw in the proof of the multiplicative Schwarz method in [12], the convergence of the sequence of functions to the eigenfunction is not obvious in some cases. For this reason in the present work the author provides another proof for multiplicative method, presents an additive version of the algorithm, and, also, extends the area of applicability for the method.

The theory developed in this paper provides an approach in which domain decomposition methods, namely the Schwarz alternating methods, both multiplicative and additive, can be used to solve spectral problems. It is shown that a discretization of the multiplicative variant of the Schwarz method is equivalent to the block coordinate relaxation method. An additive variant of the method is suitable for realization on parallel architecture.

The outline of the paper is as follows. In Section 1 we pose the problem, formulate the Schwarz alternating method, and prove its convergence. In Section 2 we provide an additive version of the algorithm. In Section 3 discretizations of the developed methods are considered.

1. Multiplicative Schwarz method. Let $\Omega$ be a bounded domain in $\mathbb{R}^{d}$ with a Lipschitz boundary and $\mathcal{L}$ be a uniformly elliptic, self-adjoint, positive definite operator. We consider the eigenvalue problem for $\mathcal{L}$ on $\Omega$ with homogeneous Dirichlet boundary conditions:

$$
\begin{aligned}
\mathcal{L} u & =\lambda u, & & \text { in } \Omega, \\
u & =0, & & \text { on } \partial \Omega .
\end{aligned}
$$

We note that the eigenvalue problems with different types of boundary conditions on some subsets of $\partial \Omega$ can be treated in a similar way but for the sake of simplicity are not described here.

Since operator $\mathcal{L}$ is positive definite then on its domain

$$
D(\mathcal{L})=\left\{u\left|u \in L^{2}(\Omega), \mathcal{L} u \in L^{2}(\Omega), u\right|_{\partial \Omega}=0\right\}
$$

we define the energy inner product [7]:

$$
[u, v] \equiv(\mathcal{L} u, v), \quad \forall u, v \in D(\mathcal{L})
$$

and corresponding energy norm $[u]=[u, u]^{1 / 2}, u \in D(\mathcal{L})$. Completing $D(\mathcal{L})$ in [•] we define the energy space $H$ of operator $\mathcal{L}$.

The Rayleigh quotient of problem (1.1) has the form [14]:

$$
R(u)=\frac{[u]^{2}}{\|u\|^{2}} .
$$

The eigenvalues of (1.1) are defined by the expressions:

$$
\begin{aligned}
& \lambda_{1}=\inf \{R(u) \mid u \in H \backslash\{0\}\}=R\left(\varphi_{1}\right), \\
& \lambda_{k}=\inf \left\{R(u) \mid u \in H \backslash\{0\},\left(\varphi_{i}, u\right)=0, i=1, \ldots, k-1\right\}=R\left(\varphi_{k}\right),
\end{aligned}
$$


where $\left\|\varphi_{k}\right\|=1, k \geq 1$. Here we assume that the eigenspace corresponding to $\lambda_{1}$ is described by only one eigenfunction $\varphi_{1}$.

Let domain $\Omega$ be represented as a union of finite number of overlapping subdomains $\Omega_{1}, \ldots, \Omega_{m}$ with the Lipschitz boundaries $\partial \Omega_{1}, \ldots, \partial \Omega_{m}$ :

$$
\Omega=\bigcup_{i=1}^{m} \Omega_{i} .
$$

We define in $H$ closed subspaces

$$
H_{i}=\left\{u \in H \mid u(x)=0, x \in \bar{\Omega} \backslash \Omega_{i}\right\}, \quad i=1, \ldots, m .
$$

The Schwarz alternating method for evaluation of the principal eigenvalue and its corresponding eigenfunction of problem (1.1) has the following form. Let the function $u^{0} \in H \backslash\{0\}$ be defined in such a way that $\lambda_{1} \leq R\left(u^{0}\right)<\lambda_{2}$. Then the sequence of functions $\left\{u^{\alpha}\right\}$ is constructed by solving the following problems:

$$
\begin{aligned}
\lambda^{n+\frac{1}{m}}= & \inf \left\{R(u) \mid u \in u^{n}+H_{1} \backslash\{0\}\right\}=R\left(u^{n+\frac{1}{m}}\right), \\
\ldots & \ldots \\
\lambda^{n+\frac{i}{m}}= & \inf \left\{R(u) \mid u \in u^{n+\frac{i-1}{m}}+H_{i} \backslash\{0\}\right\}=R\left(u^{n+\frac{i}{m}}\right), \\
\ldots & \ldots \\
\lambda^{n+\frac{m}{m}}= & \inf \left\{R(u) \mid u \in u^{n+\frac{m-1}{m}}+H_{m} \backslash\{0\}\right\}=R\left(u^{n+\frac{m}{m}}\right), \\
\lambda^{n+1}= & \lambda^{n+\frac{m}{m}}, \quad u^{n+1}=u^{n+\frac{m}{m}}, \quad n=0,1, \ldots
\end{aligned}
$$

Note that if for some $i$ we have $\lambda^{n+\frac{i}{m}}=\lambda^{n+\frac{i-1}{m}}$ then we take $u^{n+\frac{i}{m}} \equiv u^{n+\frac{i-1}{m}}$. Here by $u^{*}+H_{i}$ we denote the direct sum of the subspaces. For any $v \in u^{*}+H_{i}$ there is a representation $v=\alpha u^{*}+v_{i}$, where $v_{i} \in H_{i}$ and $\alpha \in \mathbb{R}$. Since $R(u)=R(\alpha \cdot u)$ for any nonzero constant $\alpha$, we assume that $\left\|u^{n+\frac{i}{m}}\right\|=1$ for any $n$ and $i$.

For algorithm (1.4) we can formulate the following statement.

THEOREM 1.1. Let the initial guess $u^{0} \in H \backslash\{0\}$ satisfy the inequalities $\lambda_{1} \leq$ $R\left(u^{0}\right)<\lambda_{2}$. Then the sequence $\left\{\lambda^{n}\right\}_{n=1}^{\infty}$ converges to the principal eigenvalue $\lambda_{1}$ and the sequence $\left\{u^{n}\right\}_{n=1}^{\infty}$ can be chosen in such a way that it converges in the energy norm to the corresponding eigenfunction $\varphi_{1}$, i.e.

$$
\lim _{n \rightarrow \infty} \lambda^{n}=\lambda_{1}, \quad \lim _{n \rightarrow \infty}\left[u^{n}-\varphi_{1}\right]=0 .
$$

To prove this theorem we need an auxiliary lemma.

LEMMA 1.2. Let $U$ be a subspace of $H$ and there exists a real number $b$ such that for any $u \in U$ the inequality $R(u) \geq b$ holds true. Let $\left\{u^{k}\right\}_{k=1}^{\infty} \subset U$ be a sequence that converges to some element $u^{*}$ in $L^{2}(\Omega)$ and the sequence $\left\{R\left(u^{k}\right)\right\}_{k=1}^{\infty}$ is nonincreasing and converges to b, i.e.

$$
\lim _{k \rightarrow \infty}\left\|u^{k}-u^{*}\right\|=0, \quad \lim _{k \rightarrow \infty} R\left(u^{k}\right)=b .
$$

Then $R\left(u^{*}\right)=b$. 
Proof. First, we show that $\lim _{k, m \rightarrow \infty}\left[u^{k}, u^{m}\right]-b\left(u^{k}, u^{m}\right)=0$.

Since $\forall u \in U$ we have $R(u) \geq b$ and $u^{k} \in U$ for any $k$ then $R\left(u^{k}+\alpha u^{m}\right) \geq b$ for any $\alpha \in \mathbb{R}$. It implies the inequality

$$
\alpha^{2}\left(\left[u^{m}\right]^{2}-b\left\|u^{m}\right\|^{2}\right)+2 \alpha\left(\left[u^{k}, u^{m}\right]-b\left(u^{k}, u^{m}\right)\right)+\left(\left[u^{k}\right]^{2}-b\left\|u^{k}\right\|^{2}\right) \geq 0,
$$

which holds true for any $\alpha$ only if the discriminant of the quadratic function in the left hand side is nonpositive, i.e.

$$
\left(\left[u^{k}, u^{m}\right]-b\left(u^{k}, u^{m}\right)\right)^{2}-\left(\left[u^{k}\right]^{2}-b\left\|u^{k}\right\|^{2}\right)\left(\left[u^{m}\right]^{2}-b\left\|u^{m}\right\|^{2}\right) \leq 0 .
$$

Now since $\left\{R\left(u^{k}\right)\right\}$ is nonincreasing then $\left[u^{k}\right]^{2} \geq b\left\|u^{k}\right\|^{2}$ for any $k$. Hence,

$$
\left|\left[u^{k}, u^{m}\right]-b\left(u^{k}, u^{m}\right)\right| \leq\left(\left[u^{k}\right]^{2}-b\left\|u^{k}\right\|^{2}\right)^{1 / 2}\left(\left[u^{m}\right]^{2}-b\left\|u^{m}\right\|^{2}\right)^{1 / 2} .
$$

Then $\lim _{k \rightarrow \infty}\left(\left[u^{k}\right]^{2}-b\left\|u^{k}\right\|^{2}\right)=0$ implies $\lim _{k, m \rightarrow \infty}\left[u^{k}, u^{m}\right]-b\left(u^{k}, u^{m}\right)=0$.

Using these two limits it is easy to conclude that

$$
\lim _{k, m \rightarrow \infty}\left(\left[u^{k}-u^{m}\right]^{2}-b\left\|u^{k}-u^{m}\right\|^{2}\right)=0 .
$$

Then, since $\left\{u^{k}\right\}$ is convergent in $L^{2}(\Omega)$, from (1.5) it follows that it is also convergent in $H$. Obviously, $\left\{u^{k}\right\}$ converges in $H$ to the same element $u^{*}$. Indeed, assume that $\left\{u^{k}\right\}$ converges in $H$ to some $u_{1}$ such that $\left\|u^{*}-u_{1}\right\| \neq 0$. Then $\left[u^{k}-u_{1}\right]^{2} \geq b\left\|u^{k}-u_{1}\right\|^{2}$ for any $k$. Taking the limit as $k \rightarrow \infty$ we get $0 \geq b\left\|u^{*}-u_{1}\right\|^{2}$, i.e. $\left\|u^{*}-u_{1}\right\|=0$. This is a contradiction. Thus, we conclude that $\lim _{k \rightarrow \infty}\left[u^{k}-u^{*}\right]^{2}=0$ and, finally, $\lim _{k \rightarrow \infty} R\left(u^{k}\right)=R\left(u^{*}\right)=b$.

Now we can prove Theorem 1.1.

Proof. From the variational definition of the eigenvalues [14] it follows that the sequence $\left\{\lambda^{n}\right\}_{n=0}^{\infty}$ is nonincreasing. Since it is bounded from below by $\lambda_{1}$ then it is convergent. Let $\bar{\lambda}=\lim _{n \rightarrow \infty} \lambda^{n}$. Then $\lambda_{1} \leq \bar{\lambda}<\lambda_{2}$.

Fix an integer $i=1, \ldots, m$, and consider the minimization problem for the Rayleigh quotient on the subspace $u^{n+\frac{i-1}{m}}+H_{i}$. Let $u^{n+\frac{i}{m}}$ be a solution to this problem. Then $\lambda^{n} \geq \lambda^{n+\frac{i-1}{m}} \geq \lambda^{n+\frac{i}{m}} \geq \lambda^{n+1}$. For this reason the sequence $\left\{\lambda^{n+\frac{i}{m}}\right\}_{n=0}^{\infty}$ must converge to $\bar{\lambda}$.

We remind that on each step we normalize the elements $u^{n+\frac{i}{m}}$. Hence, $R\left(u^{n+\frac{i}{m}}\right)=\left[u^{n+\frac{i}{m}}\right]^{2}$ and

$$
\lim _{n \rightarrow \infty}\left[u^{n+\frac{i}{m}}\right]^{2}=\bar{\lambda} \text {. }
$$

From (1.6) it follows that there exists some positive constant $C$ such that $\left[u^{n+\frac{i}{m}}\right]<C$ for any $n$, i.e. the sequence $\left\{u^{n+\frac{i}{m}}\right\}_{n=0}^{\infty}$ is bounded in the energy norm. Since $H$ is compactly embedded into $L^{2}(\Omega)$ this sequence is compact in $L^{2}(\Omega)$.

Now from $\left\{u^{n+\frac{1}{m}}\right\}$ we choose a subsequence $\left\{u^{n_{1}+\frac{1}{m}}\right\}$ which converges in $L^{2}(\Omega)$ to some element $u^{\frac{1}{m}}$. Then for each $i=2, \ldots, m$, from the sequence 
$\left\{u^{n_{1, \ldots, i-1)}+\frac{i}{m}}\right\}$ we choose a subsequence $\left\{u^{n_{1}, \ldots, i}+\frac{i}{m}\right\}$ which converges in $L^{2}(\Omega)$ to some element $u^{\frac{i}{m}}$.

Finally, we obtain the sequence $\left\{u^{n_{k}+\frac{i}{m}}\right\}$ which is convergent in $L^{2}(\Omega)$ for any $i=1, \ldots, m$ :

$$
\lim _{k \rightarrow \infty}\left\|u^{n_{k}+\frac{i}{m}}-u^{\frac{i}{m}}\right\|=0 .
$$

By Lemma 1.2 for all the elements $\left\{u^{\frac{i}{m}}\right\}_{i=1}^{m}$ we have the equalities

$$
R\left(u^{\frac{i}{m}}\right)=\bar{\lambda} \text {. }
$$

Now let $v_{i}$ be an arbitrary element from $H_{i}$ such that $\left[v_{i}\right]<c=$ const. Than for any $\alpha, \beta \in \mathbb{R}$ we have $R\left(\alpha u^{\frac{i-1}{m}}+\beta v_{i}\right) \geq \bar{\lambda}$. Using (1.7) from this inequality we get

$$
\beta^{2}\left(\left[v_{i}\right]^{2}-\bar{\lambda}\left\|v_{i}\right\|^{2}\right)+2 \alpha \beta\left(\left[u^{\frac{i-1}{m}}, v_{i}\right]-\bar{\lambda}\left(u^{\frac{i-1}{m}}, v_{i}\right)\right) \geq 0,
$$

which holds true for any $\alpha$ and $\beta$ only if

$$
\left[u^{\frac{i-1}{m}}, v_{i}\right]=\bar{\lambda}\left(u^{\frac{i-1}{m}}, v_{i}\right) \text {. }
$$

Now we shall show that the limit elements $u^{\frac{i}{m}}$ coincide, i.e. there exists an element $u_{1} \in H \backslash\{0\}$ such that $u^{\frac{i}{m}}=u_{1}, i=1, \ldots, m$.

Assume that there is an index $r$ such that

$$
u^{\frac{r-1}{m}} \neq u^{\frac{r}{m}}
$$

Then there exist a real number $\alpha \neq 0$ and an element $\bar{v}_{r} \in H_{r} \backslash\{0\}$ such that $R\left(u^{\frac{r-1}{m}}\right)=R\left(u^{\frac{r}{m}}\right)=R\left(\alpha u^{\frac{r-1}{m}}+\bar{v}_{r}\right)=\bar{\lambda}$. Then we have

$$
\alpha^{2}\left(\left[u^{\frac{r-1}{m}}\right]^{2}-\bar{\lambda}\left\|u^{\frac{r-1}{m}}\right\|^{2}\right)+2 \alpha\left(\left[u^{\frac{r-1}{m}}, \bar{v}_{r}\right]-\bar{\lambda}\left(u^{\frac{r-1}{m}}, \bar{v}_{r}\right)\right)+\left(\left[\bar{v}_{r}\right]^{2}-\bar{\lambda}\left\|\bar{v}_{r}\right\|^{2}\right)=0 .
$$

From (1.7) and (1.8) it follows that $\left[\bar{v}_{r}\right]^{2}=\bar{\lambda}\left\|\bar{v}_{r}\right\|^{2}$. Since $\bar{v}_{r} \neq 0$ we have $R\left(\bar{v}_{r}\right)=\bar{\lambda}$. Then, by algorithm (1.4), $\lambda^{0+\frac{i}{m}}=\lambda^{0+\frac{r}{m}}=\bar{\lambda}, i=r+1, \ldots, m$, and $\lambda^{n+\frac{i}{m}}=\lambda^{0+\frac{r}{m}}=\bar{\lambda}, n=1,2, \ldots$, and $i=1, \ldots, m$. Thus, we must have $u^{n+\frac{i}{m}} \equiv$ $u^{0+\frac{r}{m}}, n=1,2, \ldots$, and $i=1, \ldots, m$. It follows that all the limit elements $u^{\frac{i}{m}}$ must be the same: $u^{\frac{i}{m}} \equiv u^{0+\frac{r}{m}}, i=1, \ldots, m$. This is a contradiction to (1.9). Hence, assumption (1.9) is wrong and there exists $u_{1} \in H \backslash\{0\}$ such that $u^{\frac{i}{m}}=u_{1}, i=1, \ldots, m$.

Then from (1.8) it follows that $\left[u_{1}, v_{i}\right]=\bar{\lambda}\left(u_{1}, v_{i}\right)$ for any $v_{i} \in H_{i}, i=$ $1, \ldots, m$. Since $H=H_{1}+\ldots+H_{m}$, an arbitrary function $v \in H$ can be represented as $v=\sum_{i=1}^{m} v_{i}$ with $v_{i} \in H_{i}$. Thus, for any $v \in H$ we have

$$
\left[u_{1}, v\right]=\bar{\lambda}\left(u_{1}, v\right) \text {. }
$$

It implies that $u_{1}$ is an eigenfunction of problem (1.1) corresponding to eigenvalue $\bar{\lambda}$. By the variational principle for eigenvalues we have $\bar{\lambda} \equiv \lambda_{1}$ and $u_{1} \equiv \varphi_{1}$. Thus, the sequence $\left\{\lambda^{n}\right\}$ converges to $\lambda_{1}$, i.e.

$$
\lim _{n \rightarrow \infty} \lambda^{n}=\lambda_{1} .
$$


Now let us show that the entire sequence $\left\{u^{n}\right\}_{n=0}^{\infty}$ defined by (1.4) converges in the energy norm.

First, we claim that

$$
\lim _{n \rightarrow \infty} \frac{\left|\left(u^{n}, \varphi_{1}\right)\right|}{\left\|u^{n}\right\| \cdot\left\|\varphi_{1}\right\|}=1
$$

For the convenience of the presentation the proof of the claim is provided in the end of this section. Assuming this claim for the moment, we complete the proof.

For any index $n$ such that $\left(u^{n}, \varphi_{1}\right)<0$ we can take $\left(-u^{n}\right)$ instead of $u^{n}$. It is easy to see that this procedure does not violate algorithm (1.4). For such a new sequence we have

$$
\lim _{n \rightarrow \infty} \frac{\left(u^{n}, \varphi_{1}\right)}{\left\|u^{n}\right\| \cdot\left\|\varphi_{1}\right\|}=1
$$

Then it is easy to check that

$$
\begin{aligned}
{\left[u^{n}-\varphi_{1}\right]^{2}=} & {\left[u^{n}\right]^{2}+\left[\varphi_{1}\right]^{2}-2\left[u^{n}, \varphi_{1}\right]=\lambda^{n}\left\|u^{n}\right\|^{2}+\lambda_{1}\left\|\varphi_{1}\right\|^{2}-2 \lambda_{1}\left(u^{n}, \varphi_{1}\right)=} \\
& \left(\lambda^{n}-\lambda_{1}\right)\left\|u^{n}\right\|^{2}+\lambda_{1}\left(\left\|u^{n}\right\|-\left\|\varphi_{1}\right\|\right)^{2}+ \\
& 2 \lambda_{1}\left\|u^{n}\right\| \cdot\left\|\varphi_{1}\right\|\left(1-\frac{\left(u^{n}, \varphi_{1}\right)}{\left\|u^{n}\right\| \cdot\left\|\varphi_{1}\right\|}\right) .
\end{aligned}
$$

Since $\left\|\varphi_{1}\right\|=1$ and $\left\|u^{n}\right\|=1$ for all $n$, then using (1.11) and (1.12) we take the limit in (1.13) as $n \rightarrow \infty$. Then

$$
\lim _{n \rightarrow \infty}\left[u^{n}-\varphi_{1}\right]^{2}=0
$$

that completes the proof. $\square$

Now we provide the proof of the claim.

LEMMA 1.3. The sequence $\left\{u^{n}\right\}_{n=0}^{\infty}$ defined by algorithm (1.4) satisfies the following condition

$$
\lim _{n \rightarrow \infty} \frac{\left|\left(u^{n}, \varphi_{1}\right)\right|}{\left\|u^{n}\right\| \cdot\left\|\varphi_{1}\right\|}=1
$$

Proof. We shall prove that fact by contradiction. Assume that (1.15) is not true. Then there exists some subsequence $\left\{u^{n_{k}}\right\}_{k=0}^{\infty}$ such that

$$
\lim _{k \rightarrow \infty} \frac{\left|\left(u^{n_{k}}, \varphi_{1}\right)\right|}{\left\|u^{n_{k}}\right\| \cdot\left\|\varphi_{1}\right\|}=a<1 .
$$

Since $\left\{u^{n_{k}}\right\}$ is bounded in $H$ then in turn there exists a subsequence $\left\{u^{n_{k_{l}}}\right\}_{l=0}^{\infty}$ which is convergent in $L^{2}(\Omega)$. Let $u^{*} \neq 0$ be its limit, i.e. $\lim _{l \rightarrow \infty}\left\|u^{n_{k_{l}}}-u^{*}\right\|=0$. By Lemma $1.2\left\{u^{n_{k_{l}}}\right\}_{l=0}^{\infty}$ converges to $u^{*}$ in $H$ and $R\left(u^{*}\right)=\lambda_{1}$.

It implies that $u^{*}$ is the eigenfunction corresponding to the minimal eigenvalue $\lambda_{1}$ of problem (1.1). From (1.16) it follows that

$$
\frac{\left|\left(u^{*}, \varphi_{1}\right)\right|}{\left\|u^{*}\right\| \cdot\left\|\varphi_{1}\right\|}=a<1 \text {. }
$$


Using an orthogonalization we define the function $\varphi_{2}=u^{*}-\alpha \varphi_{1}$ such that $\left\|\varphi_{2}\right\|=1$ and $\left(\varphi_{1}, \varphi_{2}\right)=0$. Obviously, $R\left(\varphi_{2}\right)=\lambda_{1}$.

Thus, we get the eigenspace corresponding to $\lambda_{1}$, the basis of which contains at least two functions. That result contradicts the assumption on the size of the eigenspace corresponding to $\lambda_{1}$. Hence,

$$
\lim _{n \rightarrow \infty} \frac{\left|\left(u^{n}, \varphi_{1}\right)\right|}{\left\|u^{n}\right\| \cdot\left\|\varphi_{1}\right\|}=1
$$

REMARK 1.1. Algorithm (1.4) can be extended to the case of multiple eigenvalues, that is when the eigenspace corresponding to $\lambda_{1}$ has a finite dimension $r>1$. Instead of the elements $u^{n+\frac{i}{m}}$ in the definition of the algorithm we have to consider subspaces $E^{n+\frac{i}{m}}$ which are defined by $r$ linearly independent functions in $H$.

REMARK 1.2. Also, modified version of algorithm (1.4) can be used to find $\lambda_{p}, p>1$, and its corresponding eigenspace, provided that all the eigenspaces $E_{i}$, $i=1, \ldots, p-1$, corresponding to the eigenvalues $\lambda_{i}, i=1, \ldots, p-1$, are known. In this case instead of space $H$ in the algorithm we have to use the space

$$
H_{p}=\left\{u \mid u \in H,(u, \varphi)=0, \forall \varphi \in E_{i}, i=1, \ldots, p-1\right\} .
$$

2. Additive Schwarz method. Here we provide an additive version of algorithm (1.4) in which the computation in each subdomain can be carried out independently on each iteration.

Let the function $u^{0} \in H \backslash\{0\}$ be defined in such a way that $\lambda_{1} \leq R\left(u^{0}\right)<\lambda_{2}$. Then the sequence of functions $\left\{u^{\alpha}\right\}$ is constructed by solving the following problems:

$$
\begin{aligned}
& \lambda^{n+\frac{1}{m}}= \inf \left\{R(u) \mid u \in u^{n}+H_{1} \backslash\{0\}\right\}=R\left(u^{n+\frac{1}{m}}\right), \\
& \ldots \ldots \\
& \lambda^{n+\frac{i}{m}}= \inf \left\{R(u) \mid u \in u^{n}+H_{i} \backslash\{0\}\right\}=R\left(u^{n+\frac{i}{m}}\right), \\
& \ldots \cdots \\
& \lambda^{n+\frac{m}{m}}= \inf \left\{R(u) \mid u \in u^{n}+H_{m} \backslash\{0\}\right\}=R\left(u^{n+\frac{m}{m}}\right), \\
& \lambda^{n+1}= \inf \left\{R(u) \mid u \in\left\{u^{n+\frac{1}{m}}, \ldots, u^{n+\frac{m}{m}}\right\}\right\}=R\left(u^{n+1}\right) \\
& n=0,1, \ldots
\end{aligned}
$$

Note that the function $u^{n+1}$ is defined as a linear combination of functions $\left\{u^{n+\frac{i}{m}}\right\}_{i=1}^{m}$. Thus, the last variational problem in (2.1) is a finite dimensional one. As in the previous section if for some $i$ we have $\lambda^{n+\frac{i}{m}}=\lambda^{n}$ then we take $u^{n+\frac{i}{m}} \equiv u^{n}$. Also, we assume that $\left\|u^{n+\frac{i}{m}}\right\|=1$ for any $n$ and $i$.

For algorithm (2.1) we formulate the following statement.

THEOREM 2.1. Let the initial guess $u^{0} \in H \backslash\{0\}$ satisfy the inequalities $\lambda_{1} \leq$ $R\left(u^{0}\right)<\lambda_{2}$. Then the sequence $\left\{\lambda^{n}\right\}_{n=1}^{\infty}$ converges to the principal eigenvalue 
$\lambda_{1}$ and the sequence $\left\{u^{n}\right\}_{n=1}^{\infty}$ can be chosen in such a way that it converges in energy norm to the corresponding eigenfunction $\varphi_{1}$, i.e.

$$
\lim _{n \rightarrow \infty} \lambda^{n}=\lambda_{1}, \quad \lim _{n \rightarrow \infty}\left[u^{n}-\varphi_{1}\right]=0 .
$$

Proof. By the definition, $\left\{\lambda^{n}\right\}_{n=0}^{\infty}$ is the nonincreasing sequence which is bounded below by $\lambda_{1}$. Hence, it converges to some number $\bar{\lambda}$. Obviously, $\lambda_{1} \leq$ $\bar{\lambda}<\lambda_{2}$.

Since $\left\|u^{n}\right\|=1$ for any $n$ we have $R\left(u^{n}\right)=\left[u^{n}\right]^{2}$ and

$$
\lim _{n \rightarrow \infty}\left[u^{n}\right]^{2}=\bar{\lambda}
$$

It implies that there exists some positive constant $C$ such that $\left[u^{n}\right]<C$ for any $n$, i.e. the sequence $\left\{u^{n}\right\}_{n=0}^{\infty}$ is bounded in the energy norm. Hence, this sequence is compact in $L^{2}(\Omega)$ and we can choose a subsequence $\left\{u^{n_{k}}\right\}_{k=0}^{\infty}$ which is convergent in $L^{2}(\Omega)$ to some element $u^{*}$. Since $\lim _{k \rightarrow \infty} R\left(u^{n_{k}}\right)=\bar{\lambda}$ then by Lemma 1.2 we have $R\left(u^{*}\right)=\bar{\lambda}$.

Now we shall show that $\left(\bar{\lambda}, u^{*}\right)$ is an eigenpair of $L$.

Let $v_{i}$ be an arbitrary element from $H_{i}$ such that $\left[v_{i}\right]<c=$ const. Than for any $\alpha \in \mathbb{R}$ we have

$$
R\left(u^{n_{k}}+\alpha v_{i}\right) \geq \lambda^{n_{k}+\frac{i}{m}} \geq \lambda^{n_{k}+1} .
$$

Since $\left\{u^{n_{k}}\right\}_{k=0}^{\infty}$ is convergent in both $L^{2}(\Omega)$ and $H$, we can take the limit in (2.3) as $k \rightarrow \infty$. Then we get $R\left(u^{*}+\alpha v_{i}\right) \geq \bar{\lambda}$ or, equivalently,

$$
\alpha^{2}\left(\left[v_{i}\right]^{2}-\bar{\lambda}\left\|v_{i}\right\|^{2}\right)+2 \alpha\left(\left[u^{*}, v_{i}\right]-\bar{\lambda}\left(u^{*}, v_{i}\right)\right) \geq 0 \text {. }
$$

This inequality holds true for any $\alpha$ only if $\left[u^{*}, v_{i}\right]=\bar{\lambda}\left(u^{*}, v_{i}\right)$. As before, in (1.10), we conclude that $\left[u^{*}, v\right]=\bar{\lambda}\left(u^{*}, v\right)$ for any $v \in H$. It implies that $u^{*}$ is an eigenfunction of problem (1.1) corresponding to eigenvalue $\bar{\lambda}$. By the variational principle for eigenvalues we have $\bar{\lambda} \equiv \lambda_{1}$ and $u^{*} \equiv \varphi_{1}$. Thus, the sequence $\left\{\lambda^{n}\right\}$ converges to $\lambda_{1}$, i.e.

$$
\lim _{n \rightarrow \infty} \lambda^{n}=\lambda_{1}
$$

Using the same arguments as in the proof of Theorem 1.1 and an analogue of Lemma 1.3 for algorithm (2.1) it is easy to check that the entire sequence $\left\{u^{n}\right\}_{n=0}^{\infty}$ can be defined by (2.1) in such a way that it converges in the energy norm.

3. Matrix formulation of the Schwarz method. Now we consider a discretization of the methods described in the previous sections. Let $\Omega_{i}^{h}=$ $\bigcup_{k=1}^{M_{i}} \tau_{k}, i=1, \ldots, m$, be regular partitionings of subdomains $\Omega_{i}$ into simplices $\tau_{k}[3]$ (triangles in $\mathbb{R}^{2}$ or tetrahedra in $\mathbb{R}^{3}$ ). We assume that the triangulation of the whole domain $\Omega$ can be defined as a union of the triangulations of the subdomains, i.e. $\Omega^{h}=\bigcup_{i=1}^{m} \Omega_{i}^{h}$. 
We denote by $H^{h}$ and $H_{i}^{h}, i=1, \ldots, m$, the finite element subspaces of $H$ and $H_{i}, i=1, \ldots, m$, respectively, which consist of continuous, piece-wise linear on each simplex $\tau$ functions [3].

The finite element approximation to problem (1.1) has the following form:

$$
\begin{aligned}
& \lambda_{1}^{h}=\inf \left\{R(u) \mid u \in H^{h} \backslash\{0\}\right\}=R\left(\varphi_{1}^{h}\right), \\
& \lambda_{k}^{h}=\inf \left\{R(u) \mid u \in H^{h} \backslash\{0\},\left(\varphi_{i}^{h}, u\right)=0, i=1, \ldots, k-1\right\}=R\left(\varphi_{k}^{h}\right),
\end{aligned}
$$

where we set $\left\|\varphi_{k}^{h}\right\|=1$ for all $k$. We assume that $\lambda_{1}^{h}$ is a simple eigenvalue.

Once a basis $\left\{\psi_{i}(x)\right\}_{i=1}^{N}$ for $H^{h}$ is chosen, where $N=\operatorname{dim} H^{h}$, then (3.1) leads to an algebraic eigenvalue problem

$$
A \mathbf{u}=\lambda M \mathbf{u},
$$

where $A$ and $M$ are symmetric positive definite matrices defined by $A_{j i}=\left[\psi_{i}, \psi_{j}\right]$, $M j i=\left(\psi_{i}, \psi_{j}\right), i, j=1, \ldots, N$. We introduce the inner products by $(\mathbf{u}, \mathbf{v})_{A}=$ $(A \mathbf{u}, \mathbf{v}),(\mathbf{u}, \mathbf{v})_{M}=(M \mathbf{u}, \mathbf{v}), \mathbf{u}, \mathbf{v} \in \mathbb{R}^{N}$, and its corresponding norms by $\|\mathbf{u}\|_{A}=$ $(\mathbf{u}, \mathbf{u})_{A}^{1 / 2},\|\mathbf{u}\|_{M}=(\mathbf{u}, \mathbf{u})_{M}^{1 / 2}$, respectively. The discrete Rayleigh quotient of problem (3.2) has the form [3]:

$$
R^{h}(\mathbf{u})=\frac{\|\mathbf{u}\|_{A}^{2}}{\|\mathbf{u}\|_{M}^{2}} .
$$

Now consider in $\mathbb{R}^{N}$ the subspaces $E^{n_{i}}$ corresponding to the finite element subspaces $H_{i}^{h}, i=1, \ldots, m$, where $n_{i}=\operatorname{dim} H_{i}^{h}$. Denote by $X_{i}=\left\{\mathbf{x}_{1}^{i}, \ldots, \mathbf{x}_{n_{i}}^{i}\right\}$ the bases of subspaces $E^{n_{i}}, i=1, \ldots, m$.

The discrete analogue of the alternating Schwarz method (1.4) for evaluating the minimal eigenvalue $\lambda_{1}^{h}$ and its corresponding eigenvector $\mathbf{u}_{1}$ of problem (3.2) has the following form.

Algorithm 3.1. Let the nonzero vector $\mathbf{y}^{0} \in \mathbb{R}^{N}$ be defined in such a way that $\lambda_{1}^{h} \leq R^{h}\left(\mathbf{y}^{0}\right)<\lambda_{2}^{h}$. Then the sequence of vectors $\left\{\mathbf{y}^{k}\right\}, k=1,2, \ldots$, is constructed as follows.

1. Set $\mathbf{y}_{0}^{k}=\mathbf{y}^{k-1}$.

2. For each $i=1, \ldots, m$, solve the problems:

$$
\mu_{1}^{k, i}=\inf \left\{R^{h}(\mathbf{y}) \mid \mathbf{y} \in \operatorname{span}\left\{X_{i} ; \mathbf{y}_{i-1}^{k}\right\}, \mathbf{y} \neq 0\right\}=R^{h}\left(\mathbf{y}^{k, i}\right) .
$$

Normalize $\mathbf{y}^{k, i}$ so that $\left\|\mathbf{y}^{k, i}\right\|_{M}=1$.

3. Define $\mathbf{y}^{k}=\mathbf{y}^{k, m}$ and $\lambda^{k}=\mu^{k, m}$.

Note that the method in the given formulation is equivalent to the method of group relaxation [6] and the method of alternating subspace iteration [8].

Now we describe the discrete analogue of the additive Schwarz method (2.1).

Algorithm 3.2. Again, let the nonzero vector $\mathrm{y}^{0} \in \mathbb{R}^{N}$ be defined in such a way that $\lambda_{1}^{h} \leq R^{h}\left(\mathbf{y}^{0}\right)<\lambda_{2}^{h}$. Then the sequence of vectors $\left\{\mathbf{y}^{k}\right\}, k=1,2, \ldots$, is constructed as follows.

1. For each $i=1, \ldots, m$, solve the problems:

$$
\mu_{1}^{k, i}=\inf \left\{R^{h}(\mathbf{y}) \mid \mathbf{y} \in \operatorname{span}\left\{X_{i} ; \mathbf{y}^{k-1}\right\}, \mathbf{y} \neq 0\right\}=R^{h}\left(\mathbf{y}^{k, i}\right) .
$$


2. Define $\lambda^{k}$ and $\mathbf{y}^{k}$ solving the variational problem

$$
\lambda^{k}=\inf \left\{R^{h}(\mathbf{y}) \mid \mathbf{y} \in \operatorname{span}\left\{\mathbf{y}^{k, 1}, \ldots, \mathbf{y}^{k, m}\right\}, \mathbf{y} \neq 0\right\}=R^{h}\left(\mathbf{y}^{k}\right) .
$$

Normalize $\mathbf{y}^{k}$ so that $\left\|\mathbf{y}^{k}\right\|_{M}=1$.

The minimization problems (3.5) on step (1) of Algorithm 3.2 can be solved independently. The minimization problem (3.6) on step (2) has the dimension $m$, which can be rather small in comparison with the sizes of subdomain problems. Thus, this algorithm is suitable for parallelization.

For both algorithms 3.1 and 3.2 we formulate the following statement.

THEOREM 3.1. Let the initial guess $\mathrm{y}^{0}$ satisfy the inequalities $\lambda_{1}^{h} \leq R^{h}\left(\mathrm{y}^{0}\right)<$ $\lambda_{2}^{h}$. Then the sequence $\left\{\lambda^{k}\right\}_{k=1}^{\infty}$ converges to the minimal eigenvalue $\lambda_{1}^{h}$ and the sequence $\left\{\mathbf{y}^{k}\right\}_{k=1}^{\infty}$ can be chosen in such a way that it converges to the corresponding eigenfunction $\mathbf{u}_{1}$, i.e.

$$
\lim _{n \rightarrow \infty} \lambda^{n}=\lambda_{1}^{h}, \quad \lim _{n \rightarrow \infty} \mathbf{y}^{n}=\mathbf{u}_{1} .
$$

The proof of this statement for Algorithm 3.1 is given in [12]. Also, the main ideas of it can be found in [6] and [8].

Below we provide the proof of Theorem 3.1 for Algorithm 3.2.

Proof. From the variational definition (3.5) of the eigenvalues $\mu_{1}^{k, i}$ it follows that $\mu_{1}^{k, i} \leq \lambda^{k-1}, i=1, \ldots, m$. From (3.6) we conclude that $\lambda^{k} \leq \mu_{1}^{k, i}$ for any $i=1, \ldots, m$. Thus, the sequence $\left\{\lambda^{k}\right\}_{k=1}^{\infty}$ is nonincreasing. Since it is bounded from below by $\lambda_{1}^{h}$ then it converges to some number $\bar{\lambda}$. Obviously, $\lambda_{1}^{h} \leq \bar{\lambda}<\lambda_{2}^{h}$.

Let us consider the sequence $\left\{\mathbf{y}^{k}\right\}_{k=1}^{\infty}$. Since $\left\|\mathbf{y}^{k}\right\|_{M}=1$ then $\left\|\mathbf{y}^{\bar{k}}\right\|_{A}^{2}=\lambda^{k}$ and, consequently, $\lim _{k \rightarrow \infty}\left\|\mathbf{y}^{k}\right\|_{A}^{2}=\lambda$. It implies that there exists a positive constant $C>0$ such that $\left\|\mathbf{y}^{k}\right\|_{A}<C$ for any $k$. Then, from $\left\{\mathbf{y}^{k}\right\}_{k=1}^{\infty}$ we can choose a subsequence $\left\{\mathbf{y}^{n_{k}}\right\}_{k=1}^{\infty}$ which converges to some vector $\mathbf{y}_{1} \in \mathbb{R}^{N}$. It is easy to see that $\left\|\mathbf{y}_{1}\right\|_{M}=\left\|y^{k}\right\|_{M}=1$ and $\left\|\mathbf{y}_{1}\right\|_{A}^{2}=\lim _{k \rightarrow \infty}\left\|\mathbf{y}^{k}\right\|_{A}^{2}=\bar{\lambda}$.

Now we need to show that the pair $\left(\bar{\lambda}, \mathbf{y}_{1}\right)$ is an eigenpair of problem (3.2). For any subspace $E^{n_{i}}, i=1, \ldots, m$, arbitrary $\mathbf{v}_{i} \in E^{n_{i}}$ and $\alpha \in \mathbb{R}$ we have

$$
\frac{\left\|\mathbf{y}_{1}+\alpha \mathbf{v}_{i}\right\|_{A}^{2}}{\left\|\mathbf{y}_{1}+\alpha \mathbf{v}_{i}\right\|_{M}^{2}} \geq \bar{\lambda}
$$

which implies that

$$
\alpha^{2}\left(\left\|\mathbf{v}_{i}\right\|_{A}^{2}-\bar{\lambda}\left\|\mathbf{v}_{i}\right\|_{M}^{2}\right)+2 \alpha\left(\left(\mathbf{y}_{1}, \mathbf{v}_{i}\right)_{A}-\bar{\lambda}\left(\mathbf{y}_{1}, \mathbf{v}_{i}\right)_{M}\right) \geq 0 .
$$

This inequality holds true for any $\alpha$ only if

$$
\left(\mathbf{y}_{1}, \mathbf{v}_{i}\right)_{A}-\bar{\lambda}\left(\mathbf{y}_{1}, \mathbf{v}_{i}\right)_{M}=0 .
$$

Since for arbitrary vector $\mathbf{v} \in \mathbb{R}^{N}$ there exists a representation

$$
\mathbf{v}=\sum_{i=1}^{m} \mathbf{v}_{i}, \quad \mathbf{v}_{i} \in E^{n_{i}}, \quad i=1, \ldots, m
$$


then, taking into account (3.7), we conclude that for any $\mathbf{v} \in \mathbb{R}^{N}$ the following equality holds true: $\left(\mathbf{y}_{1}, \mathbf{v}\right)_{A}-\bar{\lambda}\left(\mathbf{y}_{1}, \mathbf{v}\right)_{M}=0$. Hence, we have

$$
\left(A \mathbf{y}_{1}-\bar{\lambda} M \mathbf{y}_{1}, \mathbf{v}\right)=0, \quad \forall \mathbf{v} \in \mathbb{R}^{N} .
$$

From (3.8) it follows that $\left(\bar{\lambda}, \mathbf{y}_{1}\right)$ is the eigenpair of problem (3.2). By the variational principle for eigenvalues we must have $\bar{\lambda} \equiv \lambda_{1}^{h}$ and $\mathbf{y}_{1} \equiv \mathbf{u}_{1}$. Thus, the sequence $\left\{\lambda^{k}\right\}$ converges to $\lambda_{1}^{h}$.

Now we have to show that the entire sequence $\left\{\mathbf{y}^{k}\right\}$ defined by Algorithm (3.2) converges to $\mathbf{u}_{1}$. It is easy to check that we can use the approach described in the proof of Theorem 1.1.

First, using the assumption on multiplicity of $\lambda_{1}^{h}$ (it is a simple eigenvalue) we can show that

$$
\lim _{k \rightarrow \infty} \frac{\left|\left(\mathbf{y}^{k}, \mathbf{u}_{1}\right)_{M}\right|}{\left\|\mathbf{y}^{k}\right\|_{M} \cdot\left\|\mathbf{u}_{1}\right\|_{M}}=1 .
$$

Then using an analogue of (1.13) for the finite dimensional norm $\|\cdot\|_{A}$ we can demonstrate that

$$
\lim _{k \rightarrow \infty}\left\|\mathbf{y}^{k}-\mathbf{u}_{1}\right\|_{A}=0
$$

that completes the proof.

REMARK 3.1. Algorithms 3.1, 3.2 can be extended to the case of multiple eigenvalues, that is when the eigenspace corresponding to $\lambda_{1}^{h}$ has a finite dimension $r>1$. Instead of the elements $\mathbf{y}^{n, i}$ in the definition of the algorithm we have to consider subspaces $E^{n, i}$ which are defined by $r$ linearly independent vectors.

REMARK 3.2. Also, modified versions of the algorithms can be used to find $\lambda_{p}^{h}, p>1$, and its corresponding eigenspace, provided that all the eigenspaces $E_{i}, i=1, \ldots, p-1$, corresponding to the eigenvalues $\lambda_{i}^{h}, i=1, \ldots, p-1$, are known. In this case instead of space $\mathbb{R}^{N}$ in the algorithms we have to use the space

$$
H_{p}^{h}=\left\{\mathbf{u} \mid \mathbf{u} \in \mathbb{R}^{N},(\mathbf{u}, \mathbf{v})=0, \forall \mathbf{v} \in E_{i}, i=1, \ldots, p-1\right\}
$$

Acknowledgment. The author is grateful to Professor Yuri Kuznetsov for initiating the interest to this problem and to Dr. S.H. Lui for a helpful discussion on the algorithm presented in [12] leading to improved version of the proof for the multiplicative algorithm.

The author also is thankful to the NSF for the financial support awarded through the Institute for Mathematics and Its Applications.

\section{REFERENCES}

[1] P. BJøRstad, Multiplicative and additive Schwarz methods: convergence in the two-domain case, in Domain decomposition methods, Philadelphia, PA, 1989, SIAM, pp. 147-159.

[2] T. F. Chan and I. Sharapov, Subspace correction multilevel methods for elliptic eigenvalue problems, in The 9th Int. Symp. on Domain Decomposition Methods for PDEs, P. Bjørstad, M. Espedal, and D. Keyes, eds., Philadelphia, PA, 1996, SIAM. (to appear). 
[3] P. Ciarlet, The Finite Element Method for Elliptic Problems, North-Holland, Amsterdam, 1978.

[4] J. Dongarra And D. Sorensen, A fully parallel algorithm for symmetric eigenvalue problem, SIAM J. Sci. Statist. Comput., 8 (1987), pp. 139-154.

[5] M. DRyja AND O. WidLund, An additive variant of the Schwarz alternating method for the case of many subregions, Technical Report 339, Department of Computer Science, Courant Institute, New York, 1987.

[6] D. Faddeev and V. Faddeeva, Computational Methods of Linear Algebra, Freeman, San Francisco, 1963.

[7] P. Grisvard, Elliptic Problems in Nonsmooth Domains, Pitman Publishing, Boston, MA, 1985.

[8] M. Kaschiev, An iterative method for minimization of the Rayleigh-Ritz functional, vol. 6 of Computational processes and systems, Nauka, Moscow, 1988, pp. 160-170. (in Russian).

[9] P. Lions, On the Schwarz alternating method. I., in The 1st Int. Symp. on Domain Decomposition Methods for PDEs, R. Glowinski, G. Golub, G. Meurant, and J. Périaux, eds., Philadelphia, PA, 1988, SIAM.

[10] S. LuI, Domain decomposition for eigenvalue problems, preprint, Hong Kong Univ. of Science and Tech., 1995.

[11] J.-C. Luo, A domain decomposition method for eigenvalue problems, in The 5th Int. Symp. on Domain Decomposition Methods for PDEs, D. Keyes, T. Chan, G. Meurant, J. Scroggs, and R. Voigt, eds., Philadelphia, PA, 1991, SIAM, pp. 306-321.

[12] S. Maliassov, An analogue of the Schwarz method for spectral problems, Numerical methods and mathematical modeling, Inst. Numer. Math., Russian Acad. Sci., Moscow, Russia, 1992, pp. 71-79. (In Russian).

[13] A. Matsokin and S. NePomnyaschikh, The Schwarz alternating method in a subspace, Soviet Mathematics, 29 (1985), pp. 78-84.

[14] S. Mikhuin, Variational Methods in Mathematical Physics, Pergamon Press, New York, 1964.

[15] A. Stathopoulos, Y. SaAd, and C. Fischer, A Schur complement method for eigenvalue problems, in The 7th Copper Mountain Conference on Multigrid Methods, NASA Conference Proceedings, NASA, 1995 . 